\title{
STRUCTURE DETECTION FROM A 3D SET OF POINTS WITH ANISOTROPIC ALPHA-SHAPES
}

\author{
Sébastien Bougleux \\ GREYC CNRS UMR 6072, Équipe Image, ENSICAEN \\ 6 Bd du Maréchal Juin, 14050 Caen Cedex, France \\ sebastien.bougleux@greyc.ensicaen.fr \\ Mahmoud Melkemi \\ Université de Haute-Alsace, LMIA - Équipe MAGE \\ 4 rue des Frères Lumière, 68093 Mulhouse Cedex, France \\ mahmoud.melkemi@uha.fr \\ Abderrahim Elmoataz \\ Université de Caen Basse-Normandie, GREYC CNRS UMR 6072 \\ 6 Bd du Maréchal Juin, 14050 Caen Cedex, France \\ abder.elmoataz@greyc.ensicaen.fr
}

June 2005

\begin{abstract}
We present a method to extract polyhedral structures from a three-dimensional set of points, even if these structures are embedded in a perturbed background. The method is based on a family of affine diagrams which is an extension of the Voronoi diagram. These diagrams, namely anisotropic diagrams, are defined by using a parameterized distance whose unit ball is an ellipsoidal one. The parameters, upon which depends this distance, control the elongation and the orientation of the associated ellipsoidal ball. The triangulations, dual to the anisotropic diagrams, have the property to connect points that are not neighbors in the Voronoi diagram. Based on these triangulations, we define a family of three-dimensional anisotropic $\alpha$-shapes. Unlike Euclidean $\alpha$-shapes, anisotropic ones allow us to detect linear and planar structures in a given direction. The detection of more general polyhedral structures is obtained by merging several anisotropic $\alpha$-shapes computed for different orientations.
\end{abstract}

Keywords: Alpha-Shapes; Computational Morphology; Delaunay Triangulation; Interpolation; Structure Detection.

\section{Introduction}

The development of range data acquisition methods which are fast, precise and inexpensive, facilitates the emergence of many problems, such as surface reconstruction and segmentation ${ }^{1,2,3,4,5}$, meshes simplification ${ }^{6}$, extraction and recognition of features ${ }^{7}$, and so on. In this article, we study the problem of detecting linear, planar and more general polyhedral structures, from a three-dimensional set of points. The existing methods, based on neighborhood graphs and related to the Voronoi diagram, are especially conceived for set of points having certain density conditions. 
When patterns are curves or surfaces, these conditions can be established explicitly ${ }^{8,9,10}$. In several situations, these methods solve with efficiency the problem of extracting a shape from a set of points. However, they are unsuited when patterns are represented by low density set of points embedded into a perturbed background. This is explained by the fact that these methods are conceptually based on density analysis and do not integrate the morphological aspect of the structures to be detected.

To deal with these cases, we combine an anisotropic analysis with the shape of the patterns that must be extracted from the set of points. The analysis based on anisotropy is employed in situations such as the detection of two-dimensional linear structures ${ }^{11}$, the reconstruction of surfaces by local interpolation ${ }^{12}$ or the generation of anisotropic meshes using curved anisotropic Voronoi diagrams ${ }^{13}$. The method we develop concerns the extraction of polyhedral structures from a threedimensional set of points, even if these structures are embedded in a perturbed background. It is based on a family of anisotropic $\alpha$-shapes, which we define as an extension of the Euclidean one ${ }^{14}$. The family of Euclidean $\alpha$-shapes is a generalization of the convex hull of a set of points $P$. It describes the shape of $P$ at several levels of detail. For a given value of $\alpha$, the $\alpha$-shape is a set of edges and triangles, of $P$. Two or three points of $P$ form an edge or a triangle if a ball of radius $\alpha$ touches them without containing any other point. The $\alpha$-shapes are sub-graphs of the Delaunay triangulation, and can be computed from the Voronoi diagram.

Our extension of $\alpha$-shapes replaces the balls of radius $\alpha$ by ellipsoidal balls, parameterized by an orientation and an elongation. These parameters control the morphological aspect of structures to be detected. The global size of the ellipsoidal balls is given by $\alpha$. When the orientation and the elongation are fixed, and $\alpha$ varies from $+\infty$ to zero, the associated anisotropic $\alpha$-shapes first detect global structures, then more linear structures appear in the direction given by the orientation of the ellipsoidal balls. If the ellipsoidal balls are sufficiently elongated or flat, the detected structures approximate parts of straight lines or planes. The significant structures are those having enough components. More generally, when the size of the ellipsoidal balls is known, polyhedral features can be extracted following these steps: first, we compute anisotropic $\alpha$-shapes in several orientations, then in each computed shape, we extract structures by deleting the non-significant ones. Finally, the obtained structures are merged.

As for $\alpha$-shapes, anisotropic $\alpha$-shapes are related to particular triangulations of the set of points and to their dual diagrams. We call them anisotropic triangulations and diagrams. They are also defined using ellipsoidal balls, and they can connect points that are not neighbors in the Delaunay triangulation. Moreover, the whole anisotropic concepts are linked to the Delaunay triangulation by an affine transformation. This allows to compute the anisotropic concepts directly from the Delaunay triangulation of the transformed set of points.

This article is organized as follows: first we recall the geometrical concepts related to $\alpha$-shapes, then we present the general anisotropic concepts, finally we apply 
anisotropic $\alpha$-shapes to detect sampled polyhedral structures.

\section{Related geometrical concepts}

\subsection{The Voronoi diagram and the Delaunay triangulation}

Let $P=\left\{p_{1}, \ldots, p_{n}\right\}$ be a finite three-dimensional set of points. Let $d_{2}(p, q)$ be the Euclidean distance between two points $p$ and $q$. The Voronoi cell $V\left(p_{i}, P\right)$ of $p_{i} \in P$ is the set of points $p \in \mathbb{R}^{3}$ such that $d\left(p, p_{i}\right) \leq d\left(p, p_{j}\right)$, for all $p_{j} \in P$. The Voronoi diagram of $P$, denoted by $V(P)$, is the set of the Voronoi cells $V\left(p_{i}, P\right)$. From a morphological point of view, $V(P)$ is generated using a unit ball as a structuring element. For two points $p$ and $q$, let $b_{q}(p)$ be the open ball, centered in $p$, and of radius $d_{2}(p, q)$. Then the Voronoi cell of $p_{i}$ corresponds to the set of points $p \in \mathbb{R}^{3}$ such that there exists an open ball $b_{p_{i}}(p)$ with $b_{p_{i}}(p) \cap P=\emptyset$.

Two points are neighbors in $V(P)$ if the intersection of their cells is not empty. The graph obtained by connecting the points of $P$ to their neighbors is the dual of $V(P)$, called the Delaunay triangulation of $P$ and noted $D(P)$. Recall that a $k$-simplex, noted $s(T)$, is a convex polytope which vertices $T$ are $k+1$ affinely independent points. A 0 -simplex is a vertex, a 1-simplex is an edge, a 2-simplex is a triangle and a 3 -simplex is a tetrahedron. Then $D(P)$ is a graph formed with $k$-simplicies, $0 \leq k \leq 3$, circumscribed to the boundary of an open ball which is empty of points of $P$. Each $k$-simplex $s(T)$ of $D(P)$ is the dual of the intersection of the $k+1$ cells $V\left(p_{i}, P\right), p_{i} \in T$.

\subsection{Euclidean $\alpha$-shapes}

Let $b(\alpha), 0<\alpha<+\infty$, be an open ball of radius $\alpha, b(\alpha)$ is a point if $\alpha=0$ and a half-space if $\alpha=+\infty$. The $\alpha$-shape of $P$, denoted by $F_{\alpha}(P)$, is the set of $k$-simplicies $s(T), k=1,2$ and $T \subset P$, such that there exists an open ball $b(\alpha)$ with $b(\alpha) \cap P=\emptyset$ and $T \subset \partial b(\alpha)$, where $\partial b(\alpha)$ is the boundary of $b(\alpha)$. The $\alpha$-shape of $P$ is a sub-graph of the Delaunay triangulation ${ }^{14}$. For $k=1$ or 2 , a $k$-simplex $s$ of $D(P)$ is an element of $F_{\alpha}(P)$ if and only if $\alpha_{\min }(s) \leq \alpha \leq \alpha_{\max }(s)$, where $\alpha_{\min }(s)$ and $\alpha_{\max }(s)$ are two positive real numbers that can be computed from $V(P)$. Indeed, $\alpha_{\min }(s)$ corresponds to the minimum distance from a vertex of $s$ to the Voronoi dual element of $s$. And $\alpha_{\max }(s)$ corresponds to the maximum distance.

Through the values of $\alpha$, the family of $\alpha$-shapes describes the set of points at several levels of detail. The behavior of this family is illustrated in Fig. 1 on a set of samples of straight lines, embedded in a perturbed background. When $\alpha=+\infty, F_{\alpha}(P)$ corresponds to the convex hull of $P$. When $\alpha$ decreases from $+\infty$ to $0, F_{\alpha}(P)$ extracts more detailed structures. Finally, when $\alpha=0, F_{\alpha}(P)$ is equal to $P$. In Fig. 1, the $\alpha$-shapes cannot extract the original structures of Fig. 1(1). This is because some points of the background are always inside the balls, of radius $\alpha$, circumscribing two successive points of a structure. In the sequel, we present a method that replaces the balls by ellipsoidal ones. Then, if the ellipsoidal balls are 


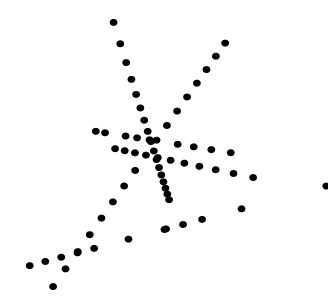

(1)

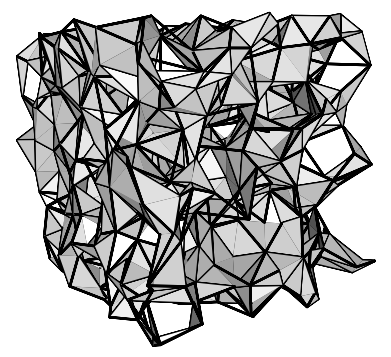

(4)

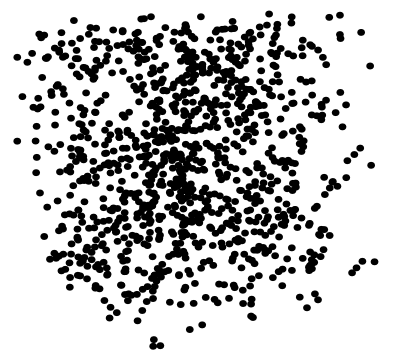

$(2)$

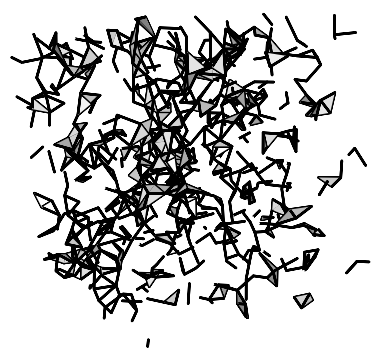

$(5)$

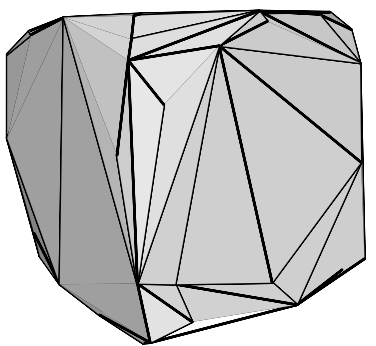

(3)

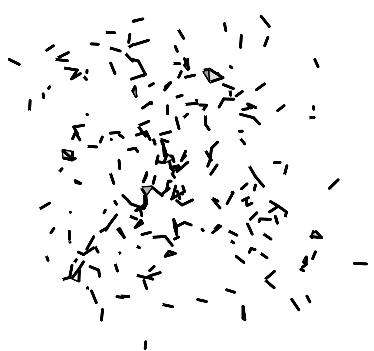

(6)

Fig. 1. Spectrum of the $\alpha$-shapes. (1) A set of points $A$, sampling 5 straight lines of $\mathbb{R}^{3}$. (2) The set $A$ is embedded in a random background, uniformly distributed. (3) $F_{+\infty}(P)=\operatorname{conv}(P)$. (4) $F_{10}(P)$. (5) $F_{5}(P)$. (6) $F_{1}(P)$.

sufficiently thin and elongated, they do not enclose any point of the background, and the suitable linear structures can be detected. The same reasoning can be performed for planar structures.

\section{Anisotropic concepts}

\subsection{Global anisotropy}

Let $R=\left(r_{i j}\right)_{1 \leq i, j \leq 3}$ be a rotation matrix. Let $E=\left\{e_{1}, e_{2}, e_{3}\right\}$ be a set of three real positive numbers. The anisotropy is formalized by the matrix:

$$
Q=\left(\begin{array}{lll}
e_{1} r_{11} & e_{1} r_{12} & e_{1} r_{13} \\
e_{2} r_{21} & e_{2} r_{22} & e_{2} r_{23} \\
e_{3} r_{31} & e_{3} r_{32} & e_{3} r_{33}
\end{array}\right) .
$$

Using this anisotropy, the distance between two points of $\mathbb{R}^{3}$ is defined by:

$$
d_{Q}(p, q)=\sqrt{(Q(p-q))^{t} Q(p-q)},
$$

where the notation $A^{t}$ represents the transpose matrix of the matrix $A$. It can be viewed as performing, a non-uniform scaling along the axes of a rotated orthogonal system, on $p$ and $q$ before taking the Euclidean distance $\left(d_{2}\right)$. Let $\tau$ be the 
transformation such that $\tau(p)=Q p$, for all $p \in \mathbb{R}^{3}$. Then from equality (2) we have:

$$
d_{Q}(p, q)=d_{2}(\tau(p), \tau(q)) .
$$

Balls associated to the distance $d_{Q}$ are ellipsoidal balls whose direction axes are given by $R^{-1}$ and elongation ratio along these axes by $E$. Let $b_{Q}(p, \alpha)$ be an open ellipsoidal ball, centered at $p$, bounded by the ellipsoid $\partial b_{Q}(p, \alpha)$ of radius $\alpha>0$. Then the semi-axes of $b_{Q}(p, \alpha)$ have lengths $\alpha \lambda_{i}$, with $\lambda_{i}=1 / e_{i}$ for $i=1,2,3$.

The effect of the transformation $\tau$, on the set of points of Fig. 1, is illustrated

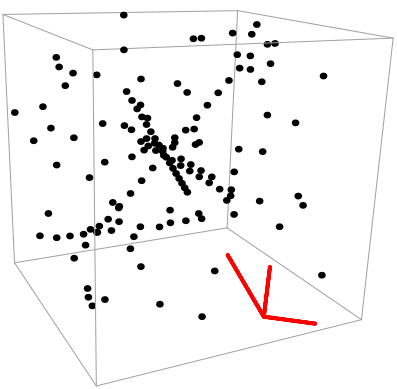

(1)

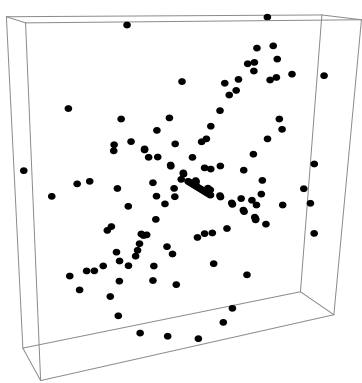

$(2)$

Fig. 2. (1) The set of points of Fig. 1 embedded in a perturbed background, and the semi-axes of a given ellipsoidal ball. (2) The set of points transformed by $\tau$.

by Fig. 2. The major axis of the ellipsoidal ball of reference is in the direction of a sampled linear structure. The points in the direction of this major axis are closer to each others, compared to their repartition in the initial set.

\subsection{Anisotropic diagrams and triangulations}

For a fixed anisotropy $Q$, let $V_{Q}\left(p_{i}, P\right)$ be the anisotropic cell of $p_{i} \in P$. It is the set of points $p \in \mathbb{R}^{3}$ such that $d_{Q}\left(p, p_{i}\right) \leq d_{Q}\left(p, p_{j}\right)$, for all $p_{j} \in P$. Based on distance $d_{Q}$, the equidistant set of points between two points is a plane. It follows that the union of the anisotropic cells defines an affine diagram ${ }^{15}$. We call it the anisotropic diagram of $P$ and we note it $V_{Q}(P)$. From a morphological point of view, $V_{Q}(P)$ is generated by ellipsoidal balls that have both the same orientation and the same elongation ratio.

The dual of $V_{Q}(P)$, obtained by connecting the points of $P$ to their neighbors, is the anisotropic triangulation of $P$, which we note $D_{Q}(P)$. It defines a set of $k$ simplicies $s(T), 0 \leq k \leq 3$, such that $s(T)$ is the dual of the intersection of the $k+1$ anisotropic cells $V_{Q}\left(p_{i}, P\right)$, for all $p_{i} \in T$. 


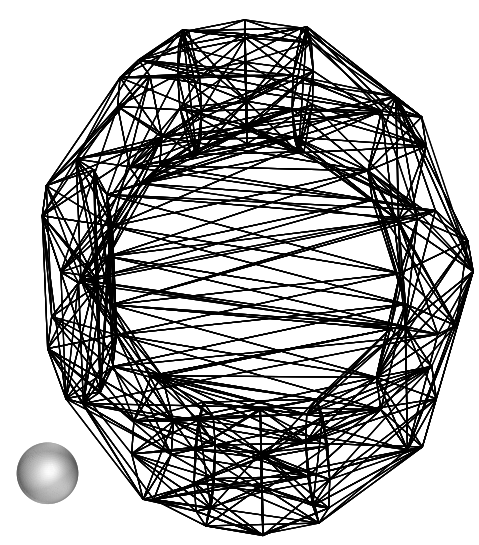

(1)

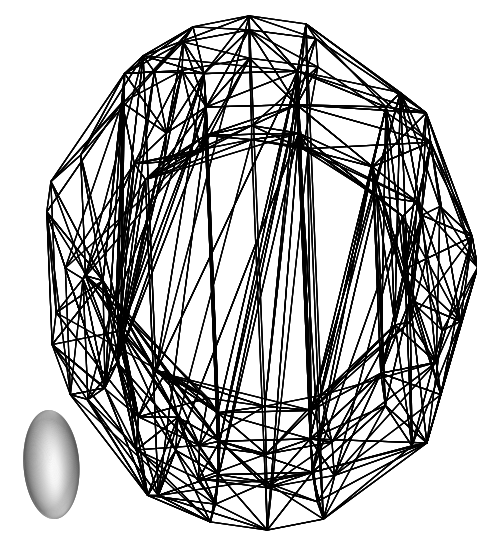

$(2)$

Fig. 3. (1) The Delaunay triangulation. (2) An anisotropic triangulation with its associated ellipsoidal ball.

While varying the orientation and the elongation ratio, the families of anisotropic diagrams and triangulations extend the Voronoi diagram and the Delaunay triangulation. Indeed, when $E=\{1,1,1\}$, ellipsoidal balls become balls, and $V_{Q}(P)$ becomes $V(P)$ (respectively $D_{Q}(P)$ becomes $D(P)$ ). An example of an anisotropic triangulation of a set of points is presented in Fig. 3(2) with its structuring element.

\subsection{Anisotropic $\alpha$-shapes}

Let $b_{Q}(\alpha)$ be an open ellipsoidal ball of radius $\alpha$ if $0<\alpha<+\infty$, a point if $\alpha=0$ and a half-space if $\alpha=+\infty$. Let $\partial b_{Q}(\alpha)$ be its boundary. And let $F_{k, \alpha, Q}(P)$ be the set of $k$-simplicies $s(T), T \subset P$, such that $b(\alpha) \cap P=\emptyset$ and $T \subset \partial b_{Q}(\alpha)$. Then, we define the anisotropic $\alpha$-shape of $P$, denoted by $F_{\alpha, Q}(P)$, as the union of the sets $F_{1, \alpha, Q}(P)$ and $F_{2, \alpha, Q}(P)$. For a given $\alpha$ and through different values of $Q$, the family of anisotropic $\alpha$-shapes extends the Euclidean ones. When $E=\{1,1,1\}$, $b_{Q}(\alpha)$ becomes $b(\alpha)$, and then $F_{\alpha, Q}(P)$ becomes $F_{\alpha}(P)$. Moreover, the anisotropic $\alpha$-shapes are sub-graphs of the anisotropic triangulations.

Observation 1 If $s$ is a simplex of $F_{\alpha, Q}(P)$ then it is a simplex of $D_{Q}(P)$.

Proof. Let $b_{Q}(c, \alpha)$ be an open ellipsoidal ball of radius $\alpha$ centered at $c$. Let $s(T)$ be a simplex of $F_{\alpha, Q}(P)$. By definition of the anisotropic $\alpha$-shape, there exists $b_{Q}(c, \alpha)$ such that $b_{Q}(c, \alpha) \cap P=\emptyset$ and $T \subset \partial b_{Q}(c, \alpha)$. This is equivalent to the following property:

$$
\forall p_{i} \in T, \forall p_{j} \in P, d_{Q}\left(c, p_{i}\right) \leq d_{Q}\left(c, p_{j}\right)
$$


From the definition of the anisotropic cells, (4) $\Leftrightarrow c \in \cap_{p_{i} \in T} V_{Q}\left(p_{i}, P\right)$. Then $\cap_{p_{i} \in T} V_{Q}\left(p_{i}, P\right) \neq \emptyset$, and thus $s(T)$ is a simplex of $D_{Q}(P)$.

\subsection{Relations between the anisotropic concepts and the Euclidean ones}

Recall that $\tau$ is the transformation represented by the matrix $Q$. Let $\tau^{-1}$ be the inverse of $\tau$ and let $\tau(P)$ be the set of points $\tau\left(p_{i}\right)$, for all $p_{i} \in P$. Let $s(T)$ be a $k$-simplex of vertices $T=\left\{p_{0}, \ldots, p_{k}\right\}$. Then $s(\tau(T))$ represents the simplex of vertices $\tau(T)=\left\{\tau\left(p_{0}\right), \ldots, \tau\left(p_{k}\right)\right\}$. The anisotropic concepts are related to the Euclidean ones by the transformation $\tau$. Therefore, given parameters $\alpha$ and $Q$, the anisotropic $\alpha$-shape can be computed from the Euclidean one. These relationships are formalized by the following observation.

Observation 2 (i) $v$ is a point of $V\left(\tau\left(p_{i}\right), \tau(P)\right)$ if and only if $\tau^{-1}(v)$ is a point of $V_{Q}\left(p_{i}, P\right)$. (ii) For $\alpha \in \mathbb{R}^{+}, s(T)$ is a simplex of $F_{\alpha, Q}(P)$ if and only if $s(\tau(T)$ ) is a simplex of $F_{\alpha}(\tau(P))$.

Proof. (i) Let $v$ be a point of $V\left(\tau\left(p_{i}\right), \tau(P)\right)$. From the definition of $V\left(\tau\left(p_{i}\right), \tau(P)\right)$, $d_{2}\left(\tau\left(p_{i}\right), v\right) \leq d_{2}\left(\tau\left(p_{j}\right), v\right)$ for all $\tau\left(p_{j}\right) \in \tau(P)$. We observe that $\tau^{-1}(\tau(p))=p$ for all $p \in \mathbb{R}^{3}$. From the equality (3), this is equivalent to $d_{Q}\left(p_{i}, \tau^{-1}(v)\right) \leq$ $d_{Q}\left(p_{j}, \tau^{-1}(v)\right)$ for all $p_{j} \in P$. This means that $\tau^{-1}(v)$ is a point of $V_{Q}\left(p_{i}, P\right)$. (ii) Let $s(T)$ be a simplex of $F_{\alpha, Q}(P)$. According to the definition of $F_{\alpha, Q}(P)$, there exists an open ellipsoidal ball $b_{Q}(c, \alpha)$ such that $b_{Q}(c, \alpha) \cap P=\emptyset$ and $T \subset \partial b_{Q}(c, \alpha)$. These properties are equivalent to the property (4). According to equality (3), we have $(4) \Leftrightarrow \forall p_{i} \in T, \forall p_{j} \in P, d_{2}\left(\tau(c), \tau\left(p_{i}\right)\right)<d_{2}\left(\tau(c), \tau\left(p_{j}\right)\right), d_{2}\left(\tau(c), \tau\left(p_{i}\right)\right)=\alpha$. So there exists an open ball $b(\tau(c), \alpha)$ such that $b(\tau(c), \alpha) \cap \tau(P)=\emptyset$ and $\tau(T) \subset \partial b(\tau(c), \alpha)$. Thus, from the definition of the Euclidean $\alpha$-shape, $s(\tau(T))$ is a simplex of $F_{\alpha}(\tau(P))$.

Based on this observation, the algorithm, which computes $F_{\alpha, Q}(P)$, works in two main steps:

- We compute $V(\tau(P))$ and its dual $D(\tau(P))$.

- For each simplex $s(\tau(T))$ of $D(\tau(P))$, if $s(\tau(T))$ is a simplex of $F_{\alpha}(\tau(P))$, we retain $s(T)$ as a simplex of $F_{\alpha, Q}(P)$.

\section{Detection of structures}

\subsection{Detection of structures in a given direction}

The anisotropic $\alpha$-shapes detect structures, like straight lines and planes. The parameters $\alpha, E$ and $R$ control the shape and the orientation of the ellipsoidal balls. If $R$ is chosen, when $\alpha$ decreases from a fixed large value and specific values of $E$ increase from one, the shape of the associated ellipsoidal ball varies from the 


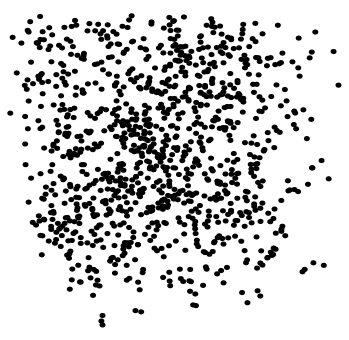

(1) $P$

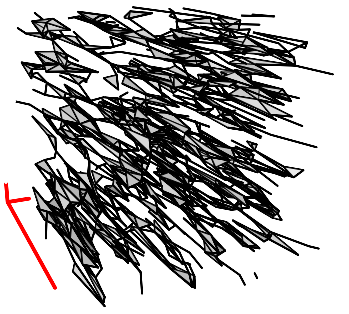

(4) $F_{3, Q}(P), \lambda_{3}=8$

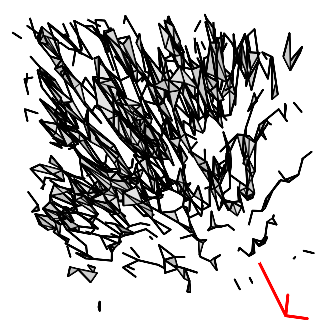

(7) $F_{6, Q}(P), \lambda_{3}=8$

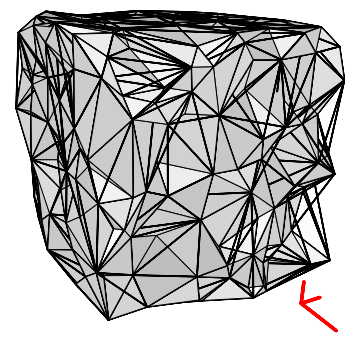

(2) $F_{20, Q}(P), \lambda_{3}=2$

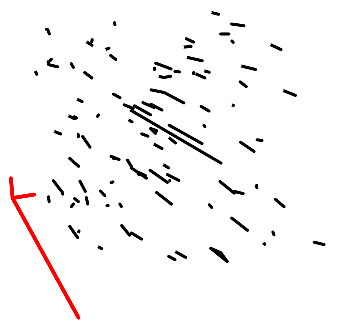

(5) $F_{1, Q}(P), \lambda_{3}=10$

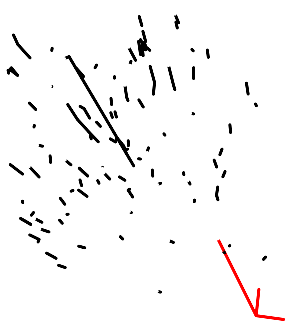

(8) $F_{1, Q}(P), \lambda_{3}=10$

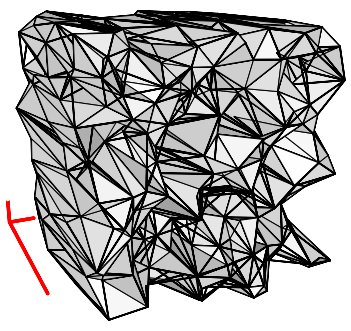

(3) $F_{10, Q}(P), \lambda_{3}=6$

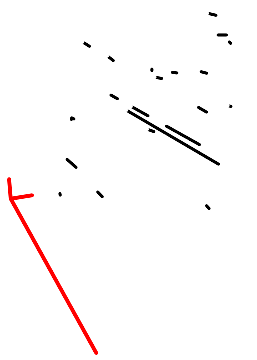

$(6) F_{0.5, Q}(P), \lambda_{3}=12$

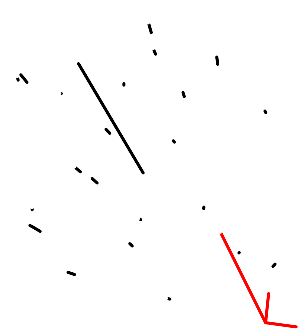

(9) $F_{0.5, Q}(P), \lambda_{3}=12$

Fig. 4. Spectrum of anisotropic $\alpha$-shapes for the sampled linear structures of Fig. 4. From (2) to (6), the orientation is fixed, and one of the three parameters $\lambda_{i}$ increases while the value of $\alpha$ decreases. The examples (7), (8) and (9) show the same results for another orientation.

ball to finer elongated balls. In these situations, the behavior of the anisotropic $\alpha$-shape is as follows: first, it detects global structures as the convex hull of the set of points. Then finer and localized structures appear gradually in the direction given by $R$ and $E$. This behavior is illustrated in Fig. 4 and Fig. 5. The example of Fig. 4 shows several anisotropic $\alpha$-shapes of the set of points of Fig. 1. Two different orientations is considered, one from Fig. 4(2) to Fig. 4(6), and the other from Fig. 4(7) to Fig. 4(9). An adequate choice of the parameters $\alpha$ and $E$ is illustrated in Fig. 4(6) and Fig. 4(9), where the suitable linear structures are detected, 


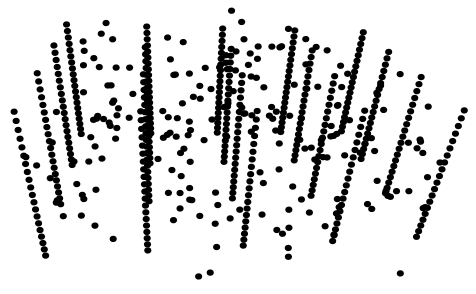

(1) $P$

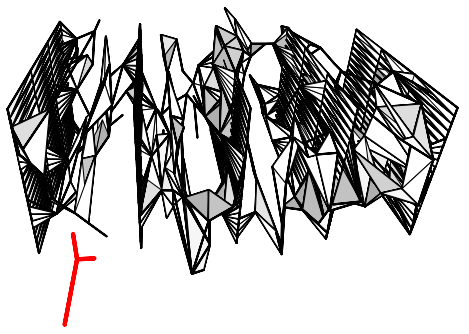

(3) $F_{15, Q}(P), \lambda_{2}=2, \lambda_{3}=6$

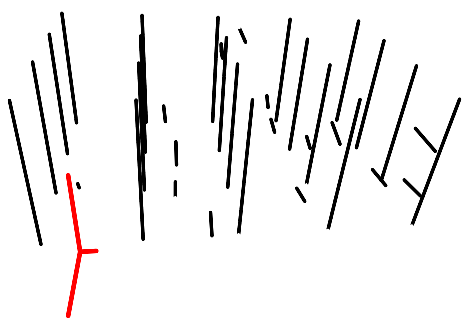

(5) $F_{2, Q}(P), \lambda_{2}=6, \lambda_{3}=16$

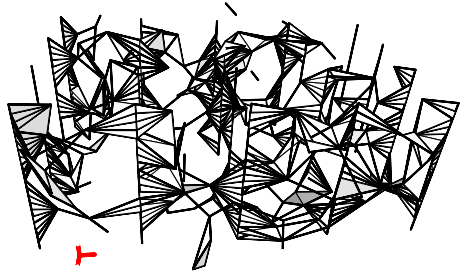

(7) $F_{30}(P)$

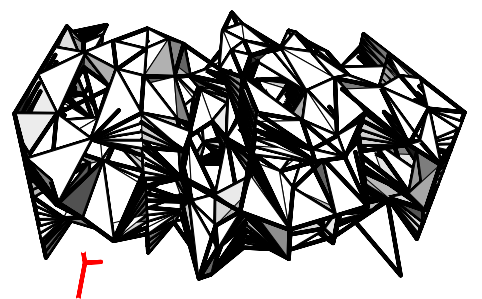

(2) $F_{30, Q}(P), \lambda_{3}=3$

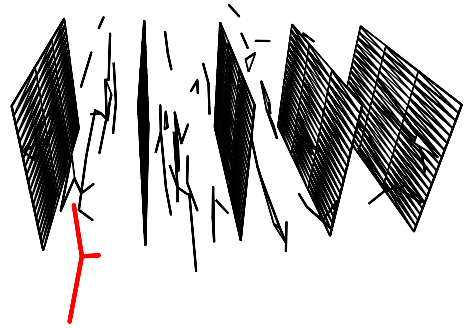

(4) $F_{6, Q}(P), \lambda_{2}=4, \lambda_{3}=12$

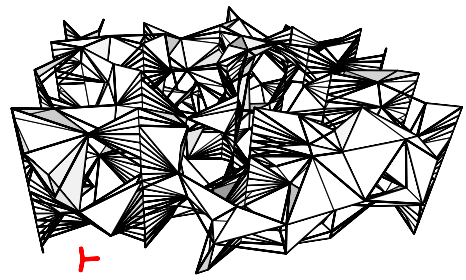

(6) $F_{40}(P)$

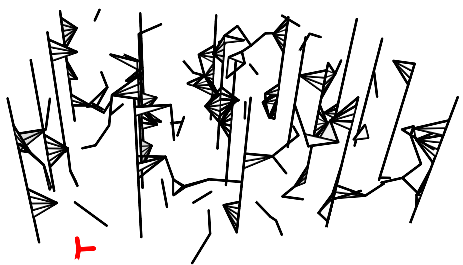

(8) $F_{20}(P)$

Fig. 5. Spectrum of anisotropic $\alpha$-shapes. (1) Sampled planes (120 points) embedded in a random perturbed background (150 points). From (2) to (5), two of the three parameters $\lambda_{i}$ increase while the orientation is fixed and the value of $\alpha$ decreases. From (6) to (8), the Euclidean $\alpha$-shapes cannot detect planar structures due to the influence of some background points.

with some non-significant ones. The same behavior is observed in Fig. 5 on samples of planar structures, embedded in a perturbed background. In Fig. 5(7), 5(8) and $5(9)$, the Euclidean $\alpha$-shapes cannot detect the planar structures. This is due to 
the interaction between the sampled points and their background neighbors. In the sequel, we analyze the relation between the pair $(\alpha, Q)$ and the detection of linear or planar structures.

\subsubsection{The case of straight lines}

Let $\left\{p_{i}, p_{i+1}, \ldots, p_{j}\right\} \subseteq P$ be a set of ordered points on a straight linear structure $L$. Let us suppose that the Euclidean distance between two consecutive sampled points is $d$. Also, let us suppose that no background points lie on $L: d_{2}(p, L) \neq 0$ for all $p \in P-\left\{p_{i}, \ldots, p_{j}\right\}$. Then, we have the following result:

Observation 3 There exists a pair $(\alpha, Q)$ such that the line segment $\left[p_{i+l}, p_{i+l+1}\right]$ is a 1-simplex of $F_{\alpha, Q}(P)$, for all $l=0, \ldots, j-i-1$.

Proof. Let $R=\left(u_{1}, u_{2}, u_{3}\right)$ be an orthonormal basis such that $L$ has direction vector collinear to $u_{1}$. Let $p_{i+l}$ and $p_{i+l+1}$ be two successive sampled points of $L$. Let $c$ be the middle of the segment $\left[p_{i+l}, p_{i+l+1}\right]$. Let us consider the ellipsoidal ball $b_{Q}(c, \alpha)$ of radius $\alpha \lambda_{1}$ in the direction of $u_{1}$, and $\alpha$ in the others $\left(\lambda_{2}=\lambda_{3}=1\right)$. Let $p$ be the point of $P \backslash\left\{p_{i+l}, p_{i+l+1}\right\}$ having the smallest distance $d_{Q}$ from $c$. Then $\left[p_{i+l}, p_{i+l+1}\right]$ is isolated from any background point if it is a simplex of $F_{1, \alpha, Q}(P)$ that satisfies the following properties: (i) $\alpha<d_{Q}(c, p)$ and (ii) $\alpha \lambda_{1}=d / 2$. The condition (i) guarantees that $q \notin b_{Q}(c, \alpha)$ and $q \notin \partial b_{Q}(c, \alpha)$, for all $q \in P \backslash\left\{p_{i+l}, p_{i+l+1}\right\}$. The condition (ii) guarantees that $\left\{p_{i+l}, p_{i+l+1}\right\} \subset$ $\partial b_{Q}(c, \alpha)$. Consequently, an adequate choice of the parameters $\alpha$ and $E$, that verify the conditions (i) and (ii) is $\lambda_{1}=d / 2 \alpha$ and $\alpha=\min \left(d / 2, d_{Q}(c, p)-\epsilon\right)$. Where $\epsilon \in\left(0, d_{Q}(c, p)\right)$ is a fixed constant introduced to satisfy the condition (i). Thus $b_{Q}(c, \alpha)$ is determined such that $\left[p_{i+l}, p_{i+l+1}\right]$ is a 1 -simplex of $F_{\alpha, Q}(P)$.

\subsubsection{The case of planar structures}

Let $P^{\prime} \subseteq P$ be a finite set of points, regularly sampled on a planar structure $L$. Let $p, p^{\prime}$ and $p^{\prime \prime}$ three points of $P^{\prime}$. As illustrated in Fig. 6, suppose that $L$ is sampled such that $d_{2}\left(p, p^{\prime}\right)=d$ in the direction given by the line $l_{1}$ and $d_{2}\left(p, p^{\prime \prime}\right)=h$ in the direction given by the line $l_{2}$, with $d \leq h$. Consider the triangle $s(T)$ of vertices $T=\left\{p, p^{\prime}, p^{\prime \prime}\right\}$. Then $s(T)$ has a unique circumscribed ellipse of least area centered at its centroid $c$, namely the Steiner ellipse of $s(T)^{16}$. Assume that no points of $P \backslash T$ lie on or inside the Steiner ellipse. Under these conditions, we have the following result:

Observation 4 There exists a pair $(\alpha, Q)$ such that the triangle $s(T)$ is a 2-simplex of $F_{\alpha, Q}(P)$.

Proof. Let $s(T)$ be the triangle of vertices $T=\left\{p, p^{\prime}, p^{\prime \prime}\right\}$. Let $R=\left(u_{1}, u_{2}, u_{3}\right)$ be a direct orthonormal basis such that $u_{1}$ is the vector $p p^{\prime}$. Let $u_{2}$ be the unit vector 


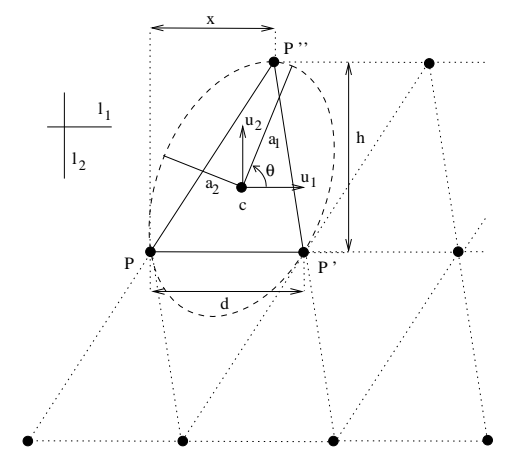

Fig. 6. A sampled planar structure and Steiner circumellipse of triangle $p p^{\prime} p^{\prime \prime}$. Its center is the triangle centroid, its semi axes are $a_{2}$ and $a_{3}$.

in the plane supported by $s(T)$ such that the angle between $u_{1}$ and $u_{2}$ is $\pi / 2$. Now consider the Steiner ellipse of $s(T)$. Let $a_{1}$ and $a_{2}$ be its major and minor semi-axes respectively. In the 2 -dimensional basis $\left(u_{1}, u_{2}\right), a_{1}$ makes an angle $\theta$ with $u_{1}$, see Fig. 6 . The parameters $a_{1}, a_{2}$ and $\theta$ can be expressed by:

$$
\begin{aligned}
a_{1} & =\frac{1}{3} \sqrt{A+2 \sqrt{B}} \\
a_{2} & =\frac{1}{3} \sqrt{A-2 \sqrt{B}} \\
\theta & =\tan ^{-1}\left(\frac{1}{2 h d}\left(\left(a^{2}-b^{2}\right)^{2}+d^{2}\left(2 d^{2}-a^{2}-b^{2}+2 \sqrt{B}\right)\right)\right)
\end{aligned}
$$

where $A=a^{2}+b^{2}+d^{2}, B=a^{4}+b^{4}+d^{4}-a^{2} b^{2}-b^{2} d^{2}-a^{2} d^{2}, a^{2}=(d-x)^{2}+h^{2}$ and $b^{2}=$ $x^{2}+h^{2}$. Let $b_{Q}(c, \alpha)$ be the ellipsoidal ball of orientation $R_{u_{3}}(\theta) R$, where $R_{u_{3}}(\theta)$ represents the rotation of an angle $\theta$ about $u_{3}$. Let $p$ be the point of $P \backslash T$ having the smallest distance $d_{Q}$ from $c$. Then $s(T)$ is isolated from any background points if it is a 2-simplex of $F_{\alpha, Q}(P)$ that satisfies the following properties: (i) $\alpha<d_{Q}(c, p)$, (ii) $\alpha \lambda_{1}=a_{1}$ and $\alpha \lambda_{2}=a_{2}$. The condition (i) guarantees that $q \notin b_{Q}(c, \alpha)$ and $q \notin \partial b_{Q}(c, \alpha)$, for all $q \in P \backslash T$. The condition (ii) guarantees that $T \subset \partial b_{Q}(c, \alpha)$. Consequently, an adequate choice of the parameters $\alpha$ and $E$, which verify the conditions (i) and (ii), is $\lambda_{1}=a_{1} / \alpha, \lambda_{2}=a_{2} / \alpha$ and $\alpha=\min \left(d_{Q}(c, p)-\epsilon, a_{1}\right)$. Where $\epsilon \in\left(0, d_{Q}(c, p)\right)$ is a small constant introduced to satisfy the condition (i). Thus $b_{Q}(c, \alpha)$ is determinated such that $s(T)$ is a 2-simplex of $F_{\alpha, Q}(P)$.

The observations 3 and 4 show that, when a linear or planar structure is regularly sampled, there exists a value $\alpha$ such that the anisotropic $\alpha$-shape detects the structure. Moreover, the detection of linear structures uses the set of 1-simplicies $F_{1, \alpha, Q}$, and the detection of planar structures uses the set of 2-simplicies $F_{2, \alpha, Q}$. 


\subsection{Removing non-significant structures}

For an adequate choice of the pair of parameters $(\alpha, Q)$, the anisotropic $\alpha$-shape detects the suitable structures. It also detects several structures joining some points of the background, see Fig. 4(6,9) and Fig. 5(4). These last structures, characterized by a low number of connected points, are called non-significants. To delete the nonsignificant structures, the sets $F_{k, \alpha, Q}(P)$ are filtered by analyzing their connected components.

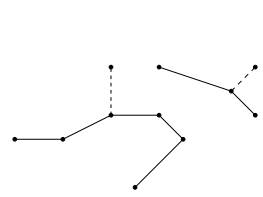

(1)

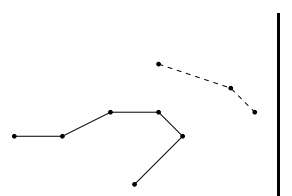

$(2)$

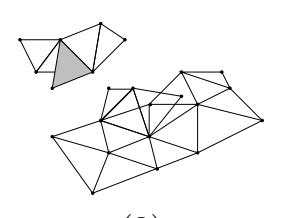

(3)

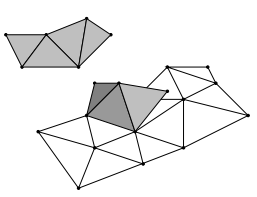

(4)

Fig. 7. $F_{1, \alpha, Q}(P)$ with $(1) n b_{c}=1$ and $(2) n b_{c}=2$ where dashed segments are deleted ; and for $F_{2, \alpha, Q}(P)$ with (3) $n b_{c}=1$ and (4) $n b_{c}=3,4$ where grey triangles are deleted.

Formally, a $k$-simplex is $h$-connected if it is incident to $h(k+1)$-simplicies. For a fixed value $k$ (1 or 2), a set of $k$-simplicies $\left\{s_{i}, s_{i+1}, \ldots, s_{j}\right\}$ is a path of length $l$ in $F_{k, \alpha, Q}(P)$ if and only if the four following properties are verified:

(i) $s_{z}$ is a $k$-simplex of $F_{k, \alpha, Q}(P)$ such that $s_{z} \cap s_{z+1} \neq \emptyset$, for all $z=i, i+1, \ldots, j-1$

(ii) for all $(k-1)$-simplex $s \subset s_{z}, s$ is 1-connected or 2-connected

(iii) there exists no $k$-simplex $s$ in $F_{k, \alpha, Q}(P)$ such that $\left\{s_{i}, \ldots, s_{j}\right\} \cup s$ verifies properties (i) and (ii)

(iv) the number of elements of $\left\{s_{i}, \ldots, s_{j}\right\}$ is $i-j+1=l$.

The property (i) guarantees that the path represents a connected structure. The property (ii) ensures that the path has no ramifications. And the properties (iii) and (iv) ensure that the length $l$ of the path is maximal.

A path is non-significant in $F_{k, \alpha, Q}(P)$ if and only if the length of the path is less than a fixed threshold $n b_{c}$. To delete the non-significant paths in a set $F_{k, \alpha, Q}(P)$, the algorithm is as follows: for $l=1, \ldots, n b_{c}$, the non-significant paths, and the 0 simplicies that become 0 -connected, are iteratively deleted. The action of the filter is illustrated in Fig. 7 for $k=1$ and 2. It can remove two kinds of non-significant structures: artifacts on the surface of a structure and small connex structures. Fig. 8 shows another example of the action of the filter on 2-simplicies.

\subsection{Detection of polyhedral structures}

For given parameters $k, \alpha$ and $E$, the family of filtered anisotropic $\alpha$-shapes of a set of points $P$, through various orientations $R$, is suitable to detect dot patterns having a polyhedral shape. In this way, we propose a general algorithm which works 

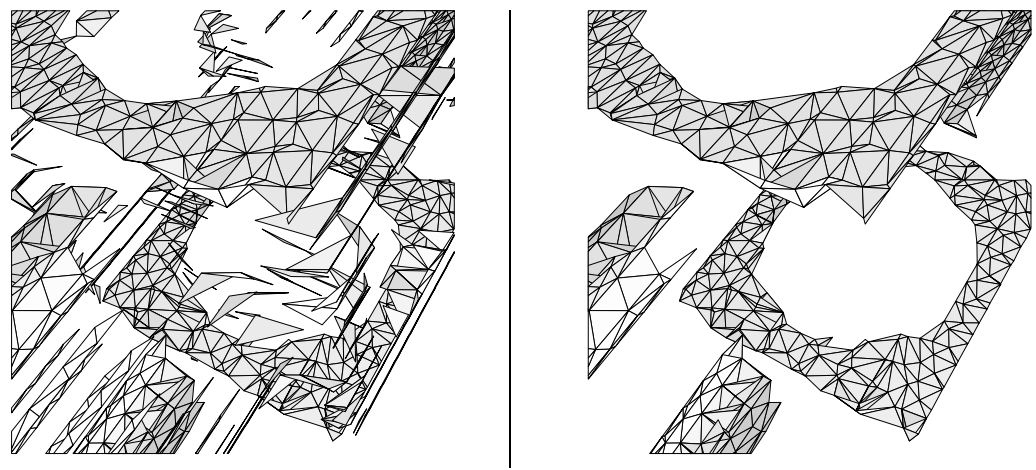

Fig. 8. Example of removing non-significant paths of 2-simplicies.

in three main steps. We use the matrix $Q$ (see equation (1)) to compute the global anisotropy on the whole set of points $P$. In $Q, R$ is represented by a composition of matrices of rotation, parameterized by Euler angles:

$$
R=R(\theta, \psi, \phi)=R_{z}(\theta) R_{x}(\psi) R_{y}(\phi),
$$

where $R_{a}(r)$ is the matrix of rotation of an angle $r$ around the $a$-axis. The coefficients of $R$ are given by:

$$
\begin{aligned}
& r_{11}=\cos \phi \cos \theta-\sin \phi \sin \theta \sin \psi \\
& r_{12}=-\sin \theta \cos \psi \\
& r_{13}=\cos \theta \sin \phi+\cos \phi \sin \theta \sin \psi \\
& r_{21}=\cos \phi \sin \theta+\cos \theta \sin \phi \sin \psi \\
& r_{22}=\cos \theta \cos \psi \\
& r_{23}=\sin \phi \sin \theta-\cos \phi \cos \theta \sin \psi \\
& r_{31}=-\sin \phi \cos \psi \\
& r_{32}=\sin \psi \\
& r_{33}=\cos \phi \cos \psi
\end{aligned}
$$

with $\theta, \phi, \psi \in(-\pi / 2, \pi / 2]$. The different orientations can be computed by several methods. In order to cover the three-dimensional space uniformly, we sample the surface of a cube by a uniform grid. Let $C$ be the box of extremities $(1,1,1)^{t}$ and $(-1,-1,0)^{t}$. The surface of the box without the $X Y$-plane, denoted by $\partial C \backslash$ $X Y$, is sampled uniformly along the axes of the Euclidean frame, with a distance $m 1$ between two successive samples. This gives a finite set of orientations $R_{m 1}=$ $\left\{R\left(0, \psi_{i}, \phi_{i}\right), i=1, \ldots, l\right\}$, where each orientation corresponds to a line $O M$ where $M$ is a sample of $\partial C \backslash X Y$, and $O$ is the origin of the Euclidean frame. This sampling method, illustrated by Fig. 9, is sufficient for extracting linear structures. In the case of planar structures, the spectrum of orientations is computed from $R_{m 1}$. For each orientation $R\left(0, \psi_{i}, \phi_{i}\right)$ in $R_{m 1}$, the angle $\theta \in(-\pi / 2, \pi / 2]$ is divided in $m 2$ 


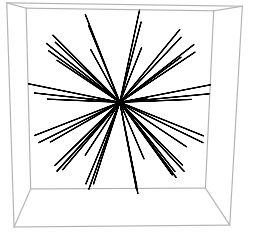

$m 1=0.6$

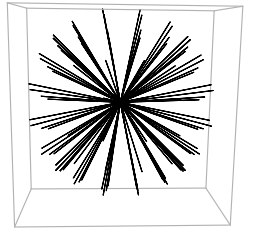

$m 1=0.4$

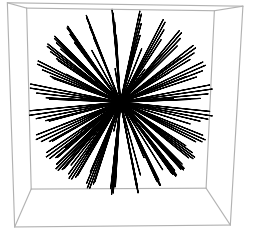

$m 1=0.3$

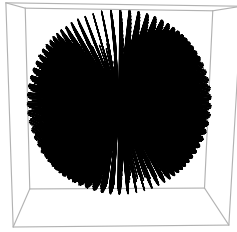

$m 1=0.1$

Fig. 9. Spectrum of orientations

angles $\theta_{j}=\theta_{j-1}+\pi / m 2$, with $j=1, \ldots, m 2$ and $\theta_{0}=-\pi / 2$. This gives a set $R_{m 1, m 2}=\left\{R\left(\theta_{j}, \psi_{i}, \phi_{i}\right), i=1, \ldots, l, j=1, \ldots, m 2\right\}$.

With the previous notations, the parameter of elongation must be given by $E=\left\{1,1,1 / \lambda_{3}\right\}$ if $k=1$, and $E=\left\{1,1 / \lambda_{2}, 1 / \lambda_{3}\right\}$ if $k=2$. In addition, let $n b_{c}>1$ be the minimum number of components of significant structures. Then, the algorithm that detects polyhedral structures is organized as follows:

- Let $A$ be $R_{m 1}$ if $k=1$ or $R_{m 1, m 2}$ if $k=2$.

- Let $F$ be a set of $k$-simplicies, initially empty. For each orientation $R(\theta, \psi, \phi)$ in $A$, the matrix $Q$ is computed with $R(\theta, \psi, \phi)$ and $E$, then $F_{k, \alpha, Q}(P)$ is computed (section 3.4) and filtered with the parameter $n b_{c}$ (section 4.2). The filtered set of $k$-simplicies is added to $F$.

- The resultant graph corresponds to the set $F$.

The first step computes the spectrum of orientations. For specific applications, this spectrum can be fixed and computed by other methods. In our experiments, we have fixed $m 1=0.3$ and $m 2=\pi / 12$. The second step corresponds to the detection process, illustrated by Fig. 10 and Fig. 11 in the case of $k=2$, and in Fig. 12 in the case of $k=1$. These synthetic examples show the detection of sampled structures in a perturbed background. The detection of planar structures in a set of sampled shapes is illustrated by Fig. 13 and Fig. 14 . The set of $k$-simplicies $F$ obtained in the third step can include some non-significant paths, introduced by the union of the filtered anisotropic $\alpha$-shapes. These bad paths can also be removed from $F$ by the filter (Fig. 14(2)).

\section{Conclusion}

In this article we showed the adequacy of a family of graphs to detect regularities in a three-dimensional set of points. This family, that we have called the anisotropic $\alpha$-shapes, is an extension of the Euclidean $\alpha$-shapes. Each anisotropic $\alpha$-shape, is generated by an ellipsoidal ball, parameterized by an elongation ratio and an orientation. From an algorithmic point of view, anisotropic $\alpha$-shapes can be computed from the Delaunay triangulation. We also proposed an algorithm, based on a spectrum of anisotropic $\alpha$-shapes, that is suitable to detect linear and planar structures 


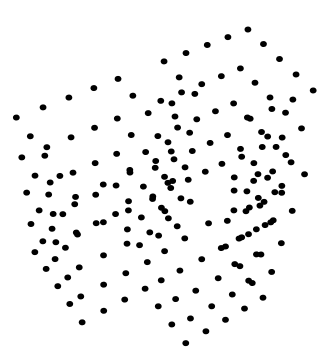

(1)

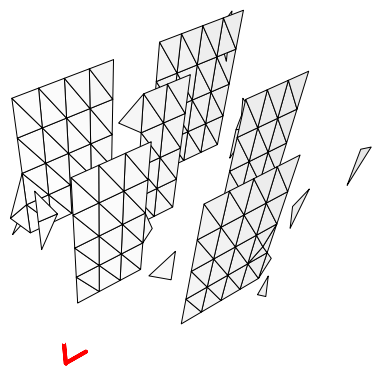

(3)

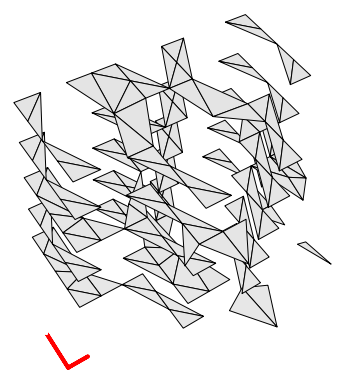

(3)

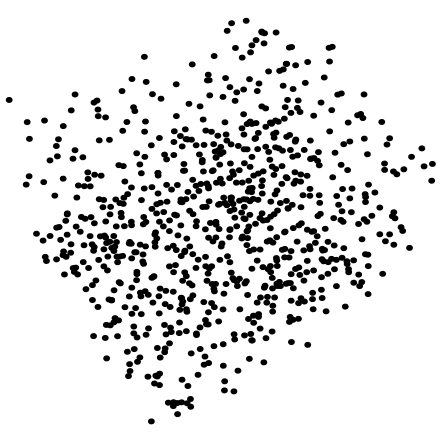

(2)

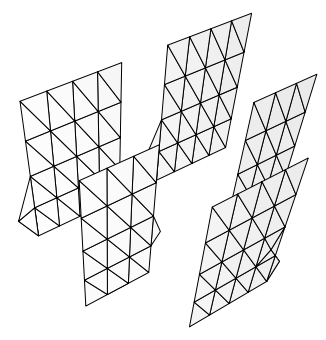

(4)

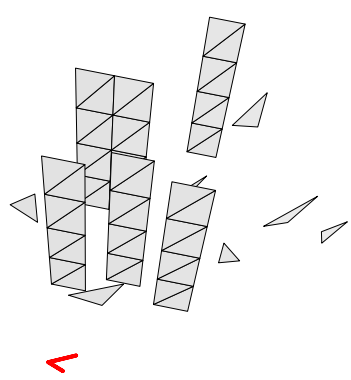

(4)

Fig. 10. Detection of planar structures with parameters $\alpha=1, \lambda_{2}=4, \lambda_{3}=8$ and $n b_{c}=20$. (1) A sampled polyhedral structure $S$ (190 points). (2) $S$ is embedded in a random perturbed background (800 points). This gives the set $P$. (3) An anisotropic $\alpha$-shape of $P$. (3) The anisotropic $\alpha$-shape, obtained in (3), is filtered. (4) and (5) show examples of anisotropic $\alpha$-shapes that are composed of non-significant structures.

in a set of points. It incorporates a method for removing non-significant details (perturbations or small parts of patterns).

The ongoing work is to construct extensions based on more complex geometric 


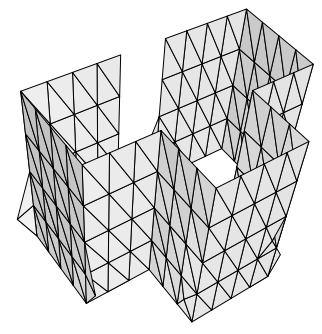

(1)

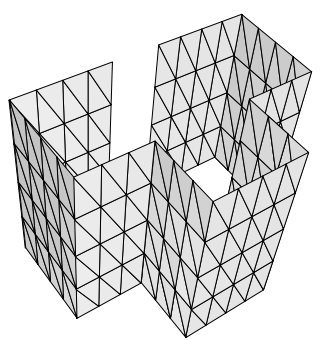

$(2)$

Fig. 11. Detection of planar structures with parameters $\alpha=1, \lambda_{2}=4, \lambda_{3}=8$ and $n b_{c}=20$. (1) The result of the algorithm, obtained from the set of points of Fig. 10. (2) The non-significant structures in (1) are deleted according to the filtering method.

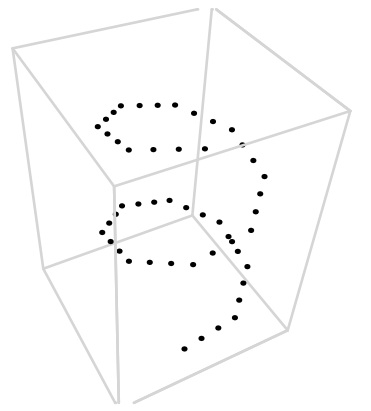

$(1)$

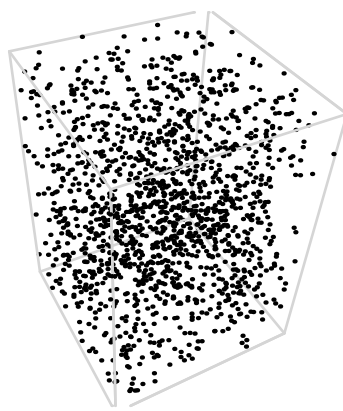

(2)

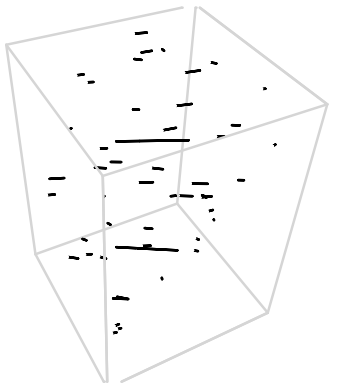

(3)

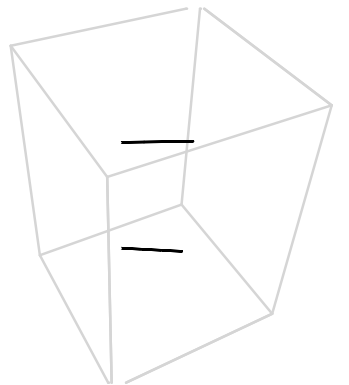

(4)

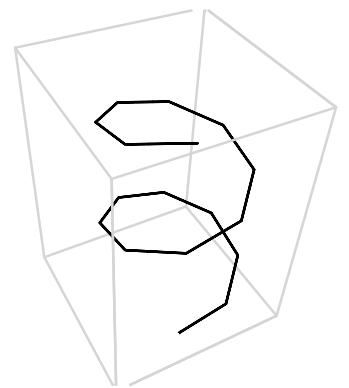

(5)

Fig. 12. Detection of linear structures $(k=1)$ with parameters $\alpha=1, \lambda_{3}=10$ and $n b_{c}=4$. (1) A set of sample points (50 points). (2) The set is embedded in a random background (1500 points). (3) An example of a suitable anisotropic $\alpha$-shape. (4) Non-significant structures in (3) have been removed. (5) The final detected structures.

elements than balls or ellipsoidal balls. These extensions will be employed to detect 


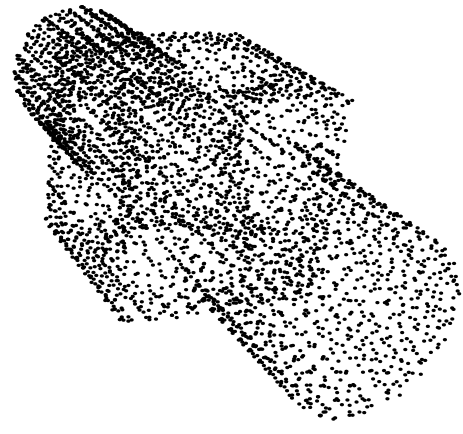

(1)

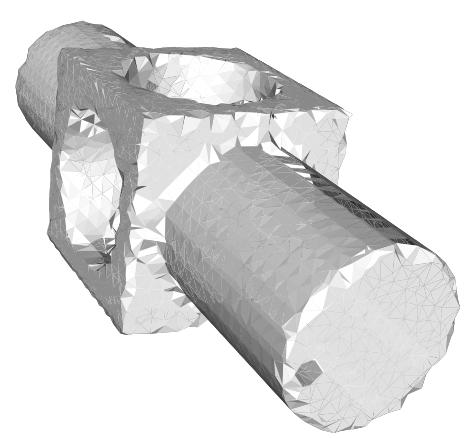

(2)

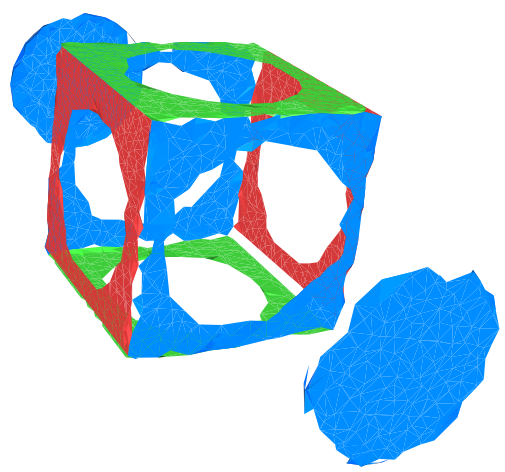

(3)

Fig. 13. Detection of planar structures. (1) A set $P$ of sampled points $(|P|=4102)$. (2) An Euclidean $\alpha$-shape of $P$. (3) The detected planar structures in $P$ with $n b_{c}=400$ and $\lambda_{2}=\lambda_{3}=20$.

patterns which are more complex than linear and planar structures. In data analysis, these methods can be used efficiently to extract patterns when the directions are known.

\section{References}

1. R. Kolluri, J.R. Shewchuk and J.F. O'Brien, "Spectral surface reconstruction from noisy point clouds", in Proc. of the Symposium on Geometry Processing, (ACM Press, 2004) p. 11-21.

2. J.C. Carr, R.K. Beatson, B.C. McCallum, W.R. Fright, T.J. McLennan and T.J. Mitchell, "Smooth surface reconstruction from noisy range data", in GRAPHITE'03: Proc. of the 1st Inter. Conf. on Computer Graphics and Interactive Techniques in Australasia and South East Asia, (ACM Press, 2003), p. 119-197.

3. Y. Ohtake, A. Belyaev and H.-P. Seidel, "Sparse surface reconstruction with adaptive partition of unity and radial basis functions", Graphical Models, Vol. 68(1) (2006) 


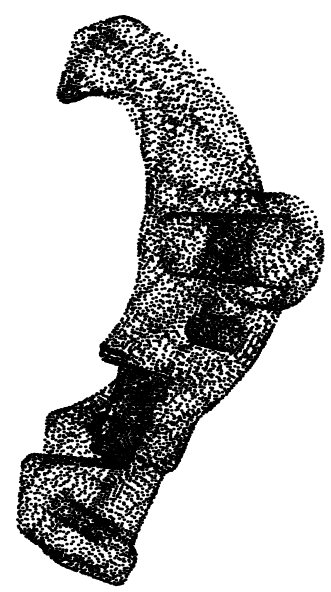

(1)

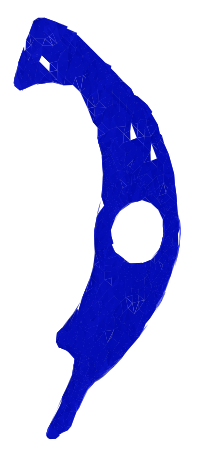

$(3)$

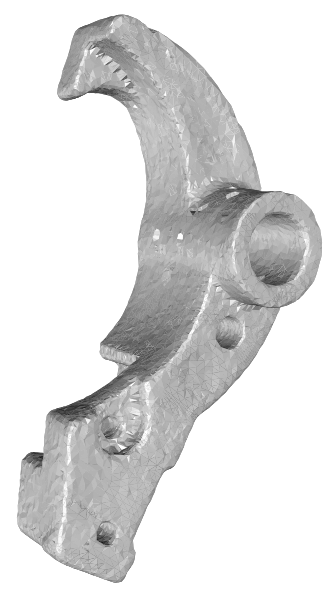

$(2)$

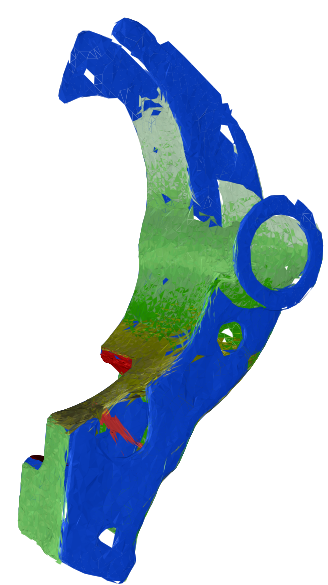

(4)

Fig. 14. Detection of planar structures with $\lambda_{2}=\lambda_{3}=10$. (1) The set of points $P(|P|=12593)$. (2) An Euclidean $\alpha$-shape of $P$. (3) The detected planar structure of maximum length (2350 components). (4) All the detected planar structures with $n b_{c}=600$.

\section{p. $15-24$.}

4. M. Djebali, M. Melkemi and N. Sapidis, "A fit-and-merge algorithm for range-image segmentation and model reconstruction", Trans. of the ASME, Journal of Computing and Information Science in Engineering, Vol. 2 (2002) p. 285-293.

5. A. Leonardis, A. Jaklic and F. Solina, "Superquadrics for segmenting and modeling range data", IEEE Trans. on Pattern Analisys and Machine Intelligence, Vol. 19(11) (1997) p. $1289-1295$.

6. J. Rossignac, "Surface simplification and 3D geometry compression", in Handbook of Discrete and Computational Geometry (2nd edition), ed. J. E. Goodman and J. O'Rourke (CRC Press, 2004) p. 1209-1240. 
7. J. O'Rourke and G.T. Toussaint, "Pattern recognition", in Handbook of Discrete and Computational Geometry (2nd edition), ed. J. E. Goodman and J. O'Rourke (CRC Press, 2004) p. 1135-1162.

8. F. Bernardini and C. Bajaj, "Sampling and Reconstructing Manifolds using AlphaShapes", in Proc. of the 9th Can. Conf. on Comp. Geometry (1997) p. 193-198.

9. J.-D. Boissonnat and S. Oudot, "Provably good sampling and meshing of surfaces", Graphical Models, Vol. 67(5) (2005) p. 405-451.

10. S-W. Cheng, T. K. Dey and E. A. Ramos, "Manifold Reconstruction from Point Samples", in Proc. of the 16th ACM-SIAM Symposium on Discrete Algorithms (2005) p. 1018-1027.

11. M. Melkemi and M. Djebali, "Elliptic diagrams: application to patterns detection from a finite set of points", Pattern Recognition Letters, Vol. 22(8) (2001) p. 835-844.

12. M. Teichmann and M. Capps, "Surface reconstruction with anisotropic density-scaled alpha shapes", in VIS'98: Proc. of the Conf. on Visualization (IEEE Computer Society Press, 1998) p. $67-72$.

13. F. Labelle and J.R. Shewchuk, "Anisotropic voronoi diagrams and guaranteed-quality anisotropic mesh generation", in SCG'03: Proc. of the 9th Annual Symp. on Computational Geometry (ACM Press, 2003) p. 191-200.

14. H. Edelsbrunner and E.P. Mücke, "Three-Dimensional Alpha Shapes", ACM Trans. on Graphics, Vol. 13(1) (1994) p. 43-72.

15. F. Aurenhammer and H. Imai", "Geometric relations among Voronoi diagrams", Geometriae Dedicata, Vol. 27 (1988) p. 65-75.

16. C. Kimberling, "Triangle Centers and Central Triangles", Congressus Numerantium, Vol. 129 (1998) p. 1-295. 